\title{
Performance results of the LHCb Silicon Tracker detector at the LHC
}

\author{
D. Esperante Pereira, ,a, B. Adeva ${ }^{a}$, A. Pazos Alvarez ${ }^{a}$, A. Gallas ${ }^{a}$, E. Perez Trigo, \\ P. Rodriguez Perez ${ }^{\mathrm{a}}$, J. Saborido ${ }^{\mathrm{a}}$, M. Needham ${ }^{\mathrm{b}}$, A. Bay ${ }^{\mathrm{b}}$, F. Blanc ${ }^{\mathrm{b}}$, J. Bressieux ${ }^{\mathrm{b}}$, \\ F. Dupertuis ${ }^{\mathrm{b}}$, V. Fave ${ }^{\mathrm{b}}$, R. Frei ${ }^{\mathrm{b}}$, N. Gauvin $^{\mathrm{b}}$, G. Haefeli ${ }^{\mathrm{b}}$, A. Keune ${ }^{\mathrm{b}}$, J. Luisier ${ }^{\mathrm{b}}$, R.

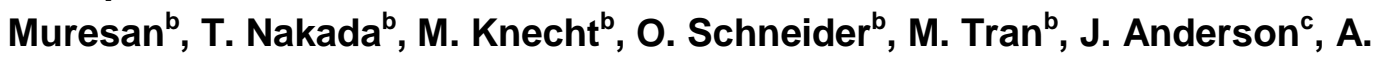 \\ Buechler $^{c}$, A. Bursche ${ }^{c}$, N. Chiapolini ${ }^{c}$, M. De Cian $^{c}$, C. Elsasser ${ }^{c}$, C. Salzmann ${ }^{c}$, S. \\ Saornil', S. Steiner ${ }^{c}$, O. Steinkamp ${ }^{c}$, U. Straumann ${ }^{c}$, J. van Tilburg ${ }^{c}$, M. Tobin $^{c}$, A. \\ Vollhardt $^{\mathrm{c}}$, O. Aquines Gutierrez ${ }^{\mathrm{d}}$, C. Bauer ${ }^{\mathrm{d}}$, M. Britsch ${ }^{\mathrm{d}}$, F. Maciuc $^{\mathrm{d}}, \mathrm{M}$. \\ Schmelling ${ }^{\mathrm{d}}$, H. Voss ${ }^{\mathrm{d}}$, V. lakovenko ${ }^{\mathrm{e}}$, O. Okhrimenko ${ }^{\mathrm{e}}$, V. Pugatch ${ }^{\mathrm{e}}$
}

\author{
${ }^{a}$ University of Santiago de Compostela, Santiago de Compostela, Spain \\ ${ }^{b}$ École Polytechnique Fédérale de Lausanne, Lausanne, Switzerland \\ ${ }^{c}$ Physik Institut der Universität Zürich, Zürich, Switzerland \\ ${ }^{d}$ Max Planck Institut für Kernphysik, Heidelberg, Germany \\ ${ }^{e}$ National Academy of Sciences, Institute for Nuclear Research, Kiev, Ukraine
}

E-mail: daniel.esperante.pereira@cern.ch

\begin{abstract}
The LHCb experiment is one of the four big experiments at the Large Hadron Collider (LHC) and it is designed to perform high-precision measurements of CP violation and search for New Physics. It is constructed as a forward single-arm spectrometer covering the polar angle 15 to 300 mrad. The Silicon Tracker (ST) is a silicon micro-strip detector designed to perform a precise measurement of the particle trajectories produced by the proton-proton interactions. It consists of two sub-detectors, the Tracker Turicensis and the Inner Tracker and covers an area of about $12 \mathrm{~m}^{2}$ in the highest occupancy region around the beam axis. Results of the detector calibration and performance using data from the LHC p-p collisions collected in the 2010 and 2011 campaigns are reported here: the time and spatial alignment of the detector was performed using data from both campaigns; studies about the intrinsic detector efficiency and resolution are also shown; recent results on the detector performance compared to the expectations will be shown as well.
\end{abstract}

10th International Conference on Large Scale Applications and Radiation Hardness of Semiconductor Detectors Firenze, Italy

July 6-8, 2011

Speaker 


\section{Introduction}

The LHCb experiment is dedicated to the study of b physics at the LHC [1]. It features excellent tracking and particle identification capabilities and is designed to perform high precision measurements of $\mathrm{CP}$ violation in $\mathrm{b}$ meson systems and rare $\mathrm{b}$ decays. Since the production of $\mathrm{b}$ pairs peaks in the forward region, along the beam axis, LHCb has been designed with a single-arm geometry that covers the polar angle of 15 to $300 \mathrm{mrad}$ (Fig. 1). The Silicon Tracker (ST) detector is part of the tracking system of LHCb and consists of two sub-detectors, the Tracker Turicensis (TT) and the Inner Tracker (IT). They both make use of silicon micro-strip technology.

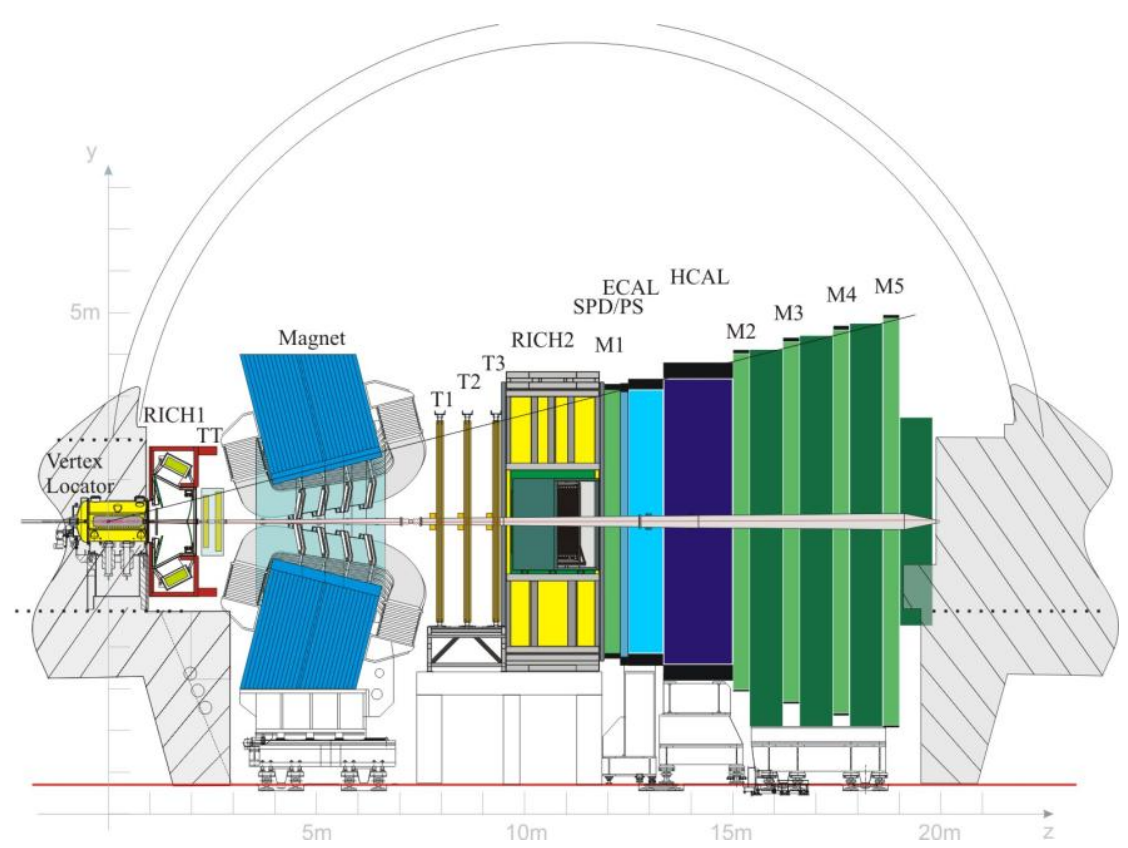

Figure 1: The LHCb experiment side-view scheme.

\section{The Silicon Tracker of LHCb}

The ST is built using silicon 'p-on-n' microstrip technology sensors from 'Hamamatsu Photonics' and features long readout strips. Detector sectors of different sizes have been constructed with 1 to 4 sensors bonded together, and thus readout strip lengths up to $38 \mathrm{~cm}$ are achieved. It covers an area of $\sim 12 \mathrm{~m}^{2}$ with $\sim 272,000$ readout channels. The detector has been designed to withstand rather high radiation doses: $5 \cdot 10^{13} 1 \mathrm{MeV}$-neutron eqv. $/ \mathrm{cm}^{2}$ after 10 years for the IT and $8 \cdot 10^{13} 1 \mathrm{MeV}$-neutron eqv./ $/ \mathrm{cm}^{2}$ for the TT. Both detectors are currently operated at a temperature of $0^{\circ} \mathrm{C}$.

The TT [1] is the first of the two sub-detectors in the downstream direction as shown in Fig. 1. It is a $\sim 150 \mathrm{~cm}$ wide and $\sim 130 \mathrm{~cm}$ height planar tracking station that is placed upstream of the magnet and covers the whole experiment acceptance. It consists of four layers (with stereo angles of $0^{\circ},+5^{\circ},-5^{\circ}$ and $0^{\circ}$ ) arranged into two half stations ('TTa' and ' $\mathrm{TTb}$ ') separated by $30 \mathrm{~cm}$ along the beam axis, as depicted in Fig. 2 (left). The sensors are assembled in 
half-modules with 7 sensors each, as shown in Fig. 3 (left), amounting to a total of 128 units, 60 in 'TTa' and 68 in 'TTb'. Electronically, each half-module is split into several readout sectors, indicated by different shadings in the figure. In the high occupancy region, close to the beam pipe, the granularity is higher with the ladders split into one, two and four sensor sectors, whereas in the outer part three and four sensor sectors are used. The readout front-end electronics (also known as hybrids) is located at the top and bottom edges of the detector to minimize the amount of dead material in the acceptance. The inner readout sectors are connected to their readout electronics via 'Kapton' interconnect cables of length $39 \mathrm{~cm}$ and $58 \mathrm{~cm}$. Finally, the sensors used in the TT are $500 \mu \mathrm{m}$ thick, with strip pitch of $183 \mu \mathrm{m}$ and sensor size of $96.4 \times 94.4 \mathrm{~mm}$, the same design as used in the CMS barrel [2]. There are a total of 143,360 readout channels.
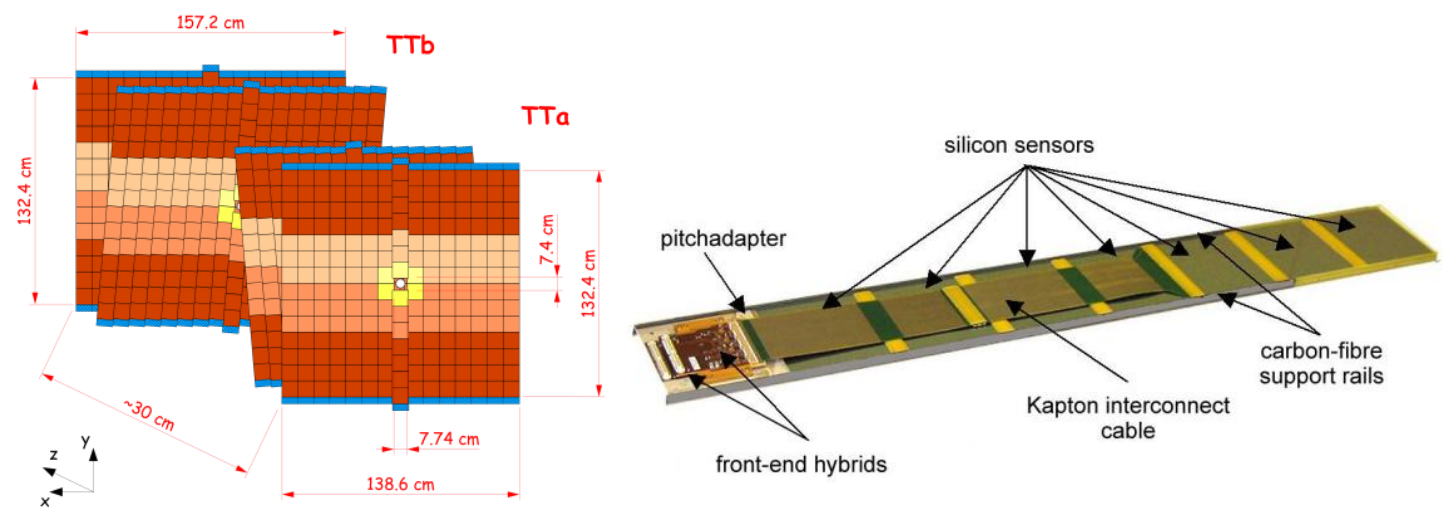

Figure 2: In the left picture, the four layers layout of the TT are shown and in blue the front-end electronics. In the right, the scheme of a half-module of the TT.

The IT covers a $125 \mathrm{~cm}$ wide and $40 \mathrm{~cm}$ height cross-shaped region (Fig. 2) in the centre of the three planar tracking stations downstream of the magnet (Fig. 1), close to the beam pipe in the region of highest particle density. Despite the fact it covers only $1.2 \%$ of the surface area, $20 \%$ of the tracks pass through it. Each of the three stations consists of four independent boxes arranged around the magnet (Fig. 3). Each box houses four layers of sensors (with stereo angles of $0^{\circ},+5^{\circ},-5^{\circ}$ and $0^{\circ}$ ). For the boxes located left and right of the beam-pipe double-sensor ladders ( $22 \mathrm{~cm}$ long in total) and sensors of $410 \mu \mathrm{m}$ thickness are used. The boxes above and below the beam-pipe consist of single-sensor modules $(11 \mathrm{~cm}$ long) of $320 \mu \mathrm{m}$ thick silicon. The readout pitch is $198 \mu \mathrm{m}$. In total there are 336 ladders and 129,024 readout channels.

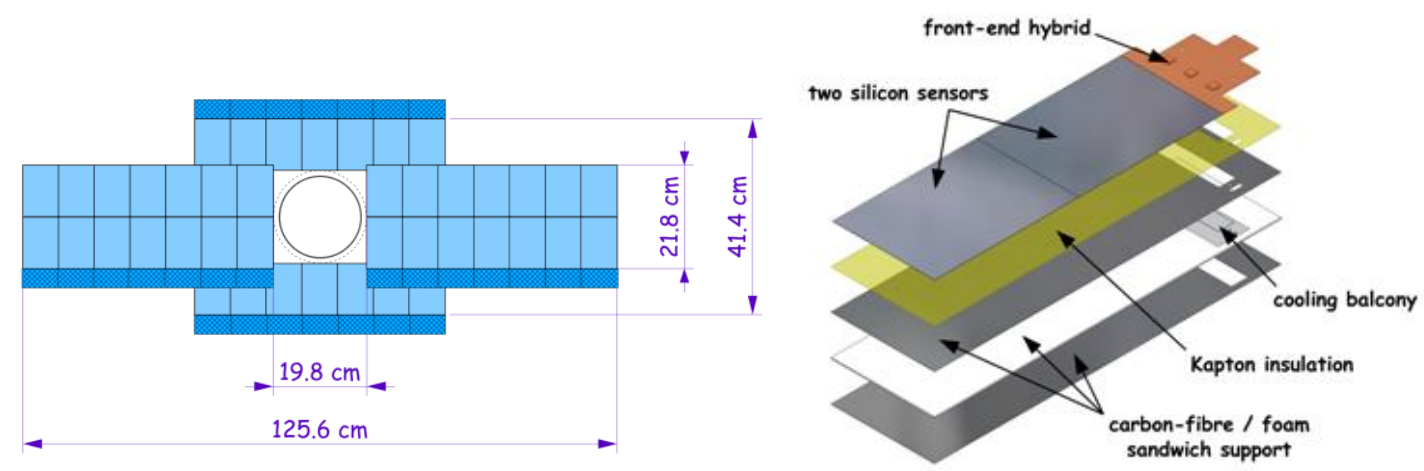

Figure 3: Layout of one IT station (left picture) and scheme of an IT module (right picture). 


\section{Detector performance}

At time of writing, more than $99 \%$ of the channels are functional: $99.7 \%$ in the TT and 98.4\% in the IT (see the maps in Fig. 4). The main sources of failures are spotted at the readout sector level and at the VCSELs of the optical readout links placed in the cavern. In the TT the problems can usually be fixed during short shutdowns thanks to the easy access to the detector and readout electronics. In the IT the situation is rather different and the malfunctioning modules may not be replaced until a long-term shutdown due to the risk the operation involves because of the difficult access to the detector elements (located very close to the Beryllium beam pipe). Nevertheless, dead VCSELS in the IT can be replaced during some shutdowns since the access is not as difficult as for the detector boxes.

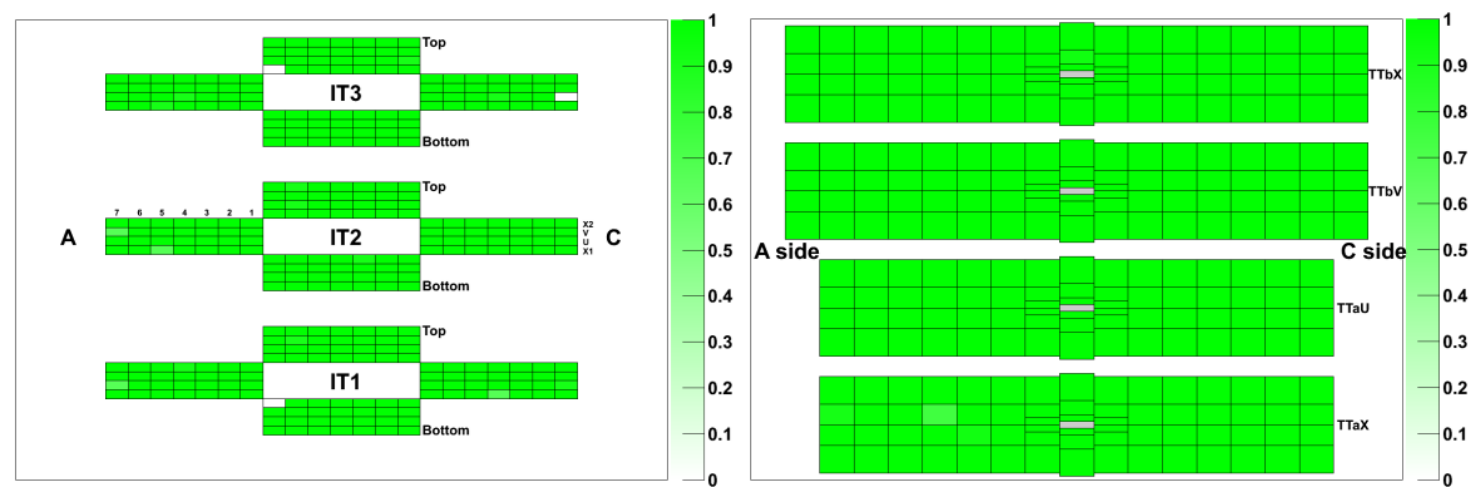

Figure 4: IT (left picture) and TT (right picture) showing the map of functional channels at June 2010.

\subsection{Time Alignment}

The readout electronics is continuously sampling the readout strips at $40 \mathrm{MHz}$ synchronous to the LHC bunch-crossing rate, so it is mandatory that the readout electronics is time aligned to the particles crossing so the detector signals are sampled in the peak (see Fig.5 left picture). Collision events are used to time align the detector with respect to the LHC collisions. An internal time alignment of the different detector readout sectors is needed to account for different lengths of cables and different time of flight for particles passing through each station. To find the optimal timing different data runs with collision events were collected for different clock delay phases in steps of $6.5 \mathrm{~ns}$. The most probable value (MPV) of the charge collection distribution was determined by fitting a Landau convolved with a Gaussian for each delay setting and for groups of readout sectors corresponding to one front-end service box. With the different MPVs corresponding to the different delays the peak of the pulse-shape was determined by fitting the expected front-end pulse-shape so the optimal timing delay can be determined (Fig. 5). After this procedure the detector was time aligned with a precision better than $1 \mathrm{~ns}$.

This procedure needs to be performed from time to time in order to account for variations in the pulse-shape timing caused by changes in the operational conditions of the detector such us working temperature and bias voltage or even by timing drifts in other parts of the LHCb timing and trigger system. 

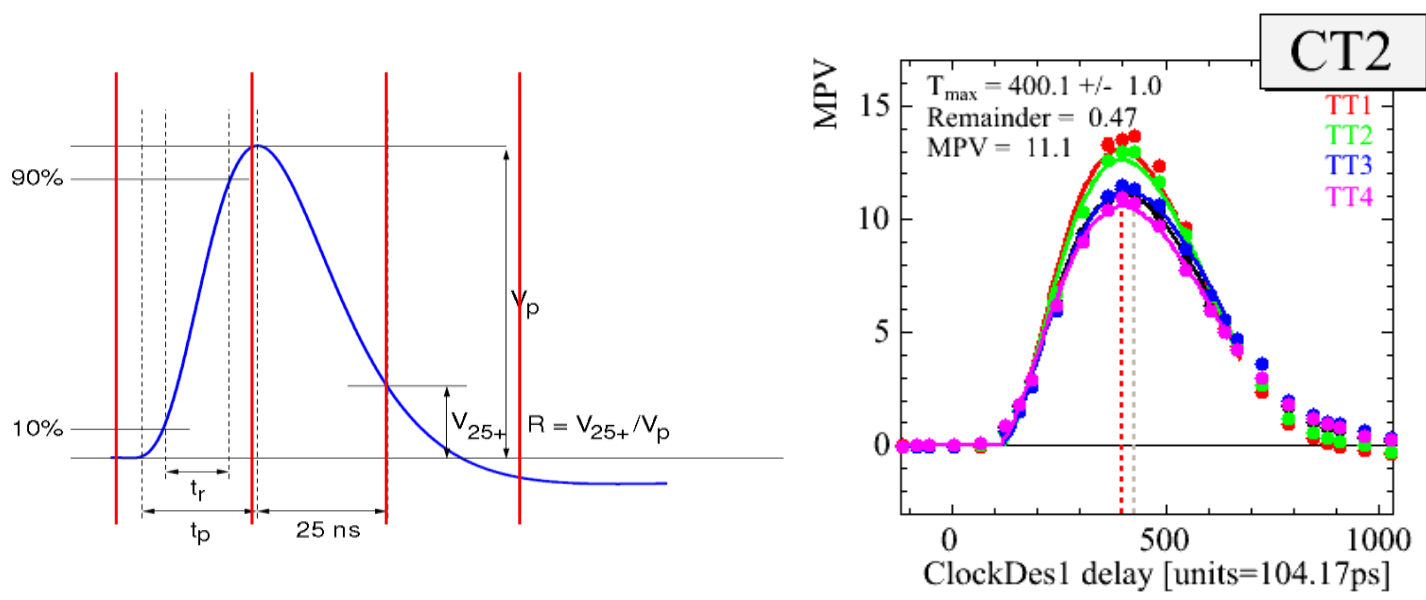

Figure 5: In the left picture an ideal pulse-shape depicted in blue with vertical red lines showing the clock sampling point at an optimal timing. In the right, reconstruction of the pulse-shape for different sector sizes in the TT.

\subsection{Signal-to-Noise (S/N) performance}

After time alignment, the detector $\mathrm{S} / \mathrm{N}$ performance has been studied. To do this study, hits from reconstructed tracks with momentum greater than $5 \mathrm{GeV}$ are used to ensure that the analysis is performed with real signals. Fig. 6 shows an example of the S/N obtained for one IT sector. A fit of a Landau convolved with a Gaussian is used to obtain the MPV for the different readout sectors both in IT and TT. Figure 7 (left) shows the S/N obtained for the individual IT short and long ladders getting as result $\sim 16.5$ and $\sim 17.5$ respectively. There is a second peak for the short ladders around $\mathrm{S} / \mathrm{N} \sim 20$ because during module production some short ladders were constructed with $410 \mu \mathrm{m}$ thick sensors rather than $320 \mu \mathrm{m}$. For the TT ladders, the $\mathrm{S} / \mathrm{N}$ is in the range 13-15 depending on the capacitance of the readout sector. These differences in capacitance are the result of grouping several modules together to assemble long strips and also due to the Kapton interconnect cables used to read out the internal modules from the readout hybrids located in the detector periphery. This is why in Fig 6 (right) the TT3 readout sector (made of 3 silicon sensors) show higher capacitance, and hence lower S/N, than the TT4.

\subsection{Spatial alignment and hit resolution}

Collision data was also used to perform the spatial alignment of the detector. The method used consists of a global $\chi^{2}$ minimization based on a Kalman filter track fit residual and is described in [3]. "Long tracks" which pass through the whole LHCb tracking system were used to align TT and IT. In addition, to ease and improve the alignment precision, a standalone track reconstruction in the three tracking stations downstream of the magnet was used in the IT. For each detector element up to six degrees of freedom can be aligned (three translations and three rotations), but for some of them the sensitivity is so low that they are left fixed. For each detector element the unbiased residuals of hits (ie, the hit is no part of the track used to calculate the residual) with respect to the reconstructed tracks are calculated. From these residual distributions a mean and a rms values are obtained, each providing valuable information of different aspects of the detector. 
Histograms for IT and TT with the means of the residual distributions of each readout sector are shown in Fig. 8. When the statistics is high enough (which is the case) the mean of the residual distribution per readout sector should be zero if the alignment was perfect, and in consequence the histogram of all these mean values should be delta at zero. However, this is not what was obtained and the plots of Fig. 8 show distributions with a sigma of $\sim 11 \mu \mathrm{m}$ for IT and $\sim 18 \mu \mathrm{m}$ for TT. This is a clear indication of the existence of some sources of misalignment that need still to be improved.

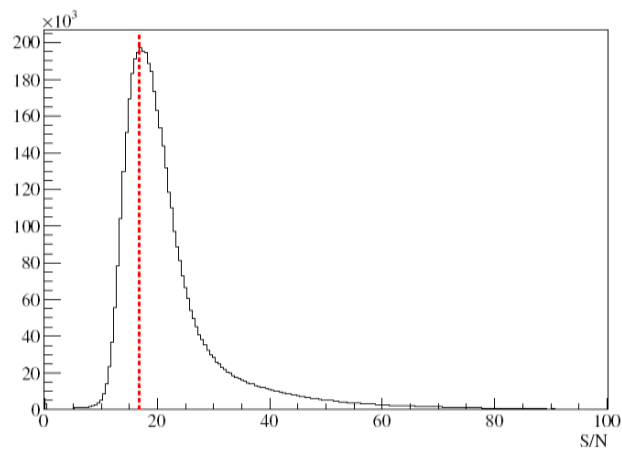

Figure 6: Fitted signal to noise ratio for an IT sector.
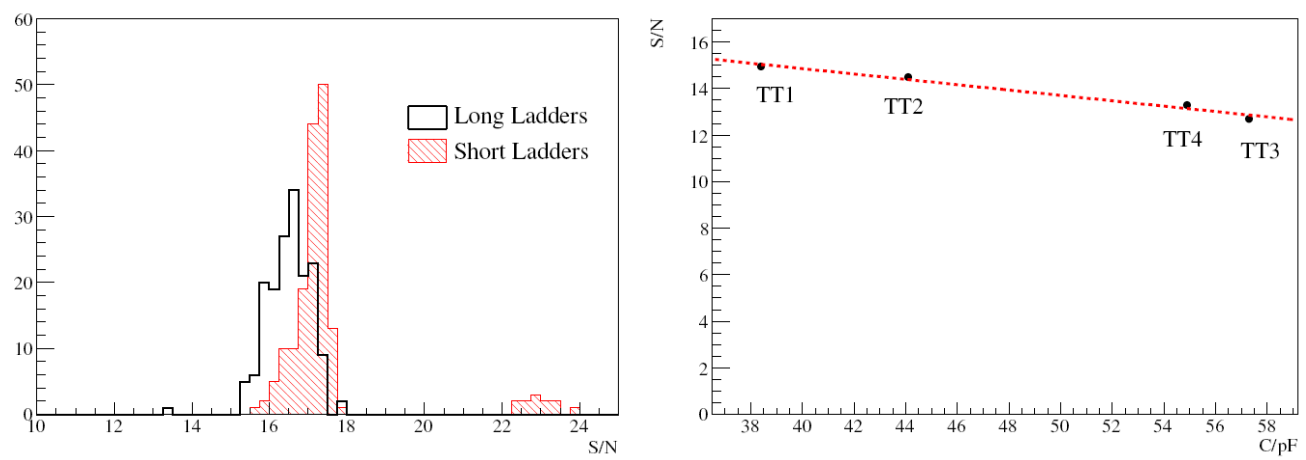

Figure 7: IT (left) and TT (right) S/N ratios.

Putting together the residual distributions corresponding to all readout sectors we obtain information about the detector hit resolution. According to the results shown in Fig. 9, the resolution for IT is $\sim 58 \mu \mathrm{m}$ (where the strip pitch is $190 \mu \mathrm{m}$ ) and for TT $\sim 62 \mu \mathrm{m}$ (strip pitch of $183 \mu \mathrm{m})$. The plots also show a discrepancy with respect to Monte Carlo, which is known to come from some difference in the gain and from the status of the alignment.
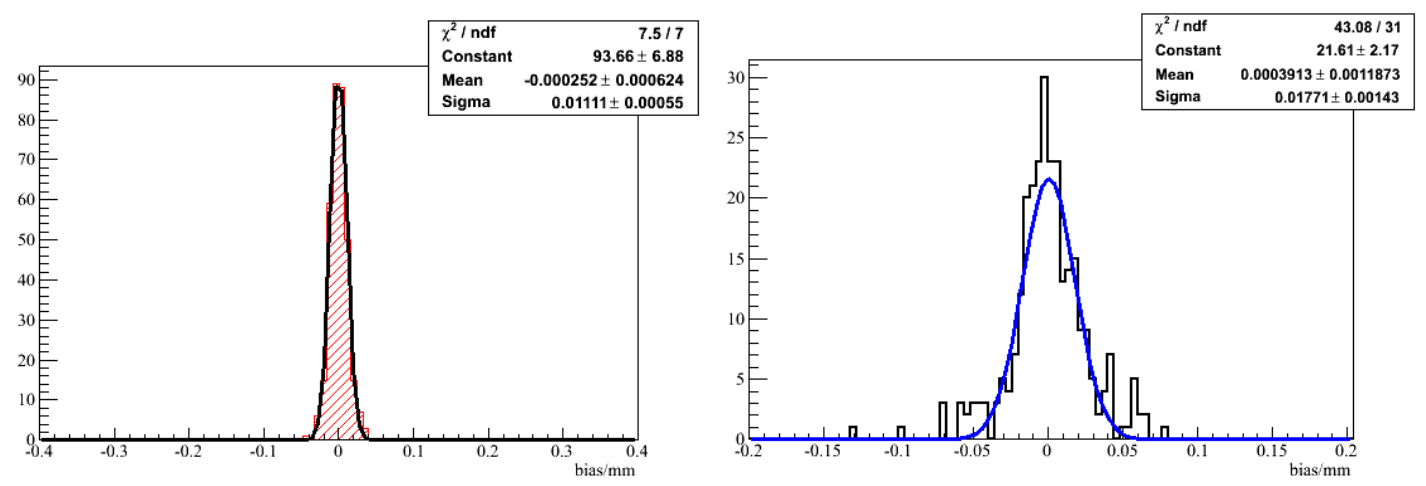

Figure 8: Histograms of the mean of the residuals per readout sector for IT (left) and TT (right). 

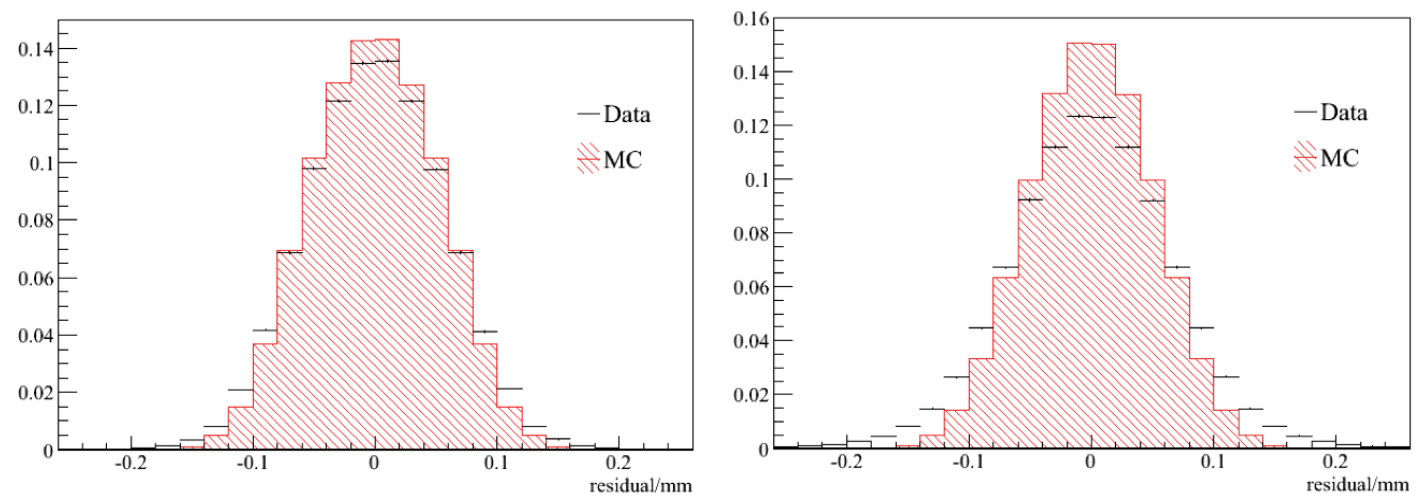

Figure 9: IT (left) and TT (right) unbiased residuals distribution, compared to the expectations from MC.

\subsection{Hit efficiency}

To assess the intrinsic detector efficiency high momentum long tracks $(\mathrm{P}>10 \mathrm{GeV})$ with a tight cut in the $\chi^{2}$ track fit are used. In addition, "events" with too many hits in a search window around the track are also discarded in order to reject possible ghost tracks. For each layer where a hit is expected, hits are searched for in a window around the track (1 mm for IT and $2.5 \mathrm{~mm}$ for TT). The efficiency is defined as the ratio of the number of found hits to the number of expected hits. It was measured to be $99.3 \%$ for IT and $99.7 \%$ for TT. The noise cluster rate was below $10^{-5}$.

\subsection{Radiation damage}

Radiation damage is a critical issue in silicon detectors. The performance of the sensors degrades significantly with radiation dose if they are not being operated in proper conditions. Therefore, monitoring of the environmental working parameters and leakage currents is advisable, particularly for the most exposed sensors, i.e. those closest to the beam pipe.

Currently, the radiation damage is monitored using the leakage currents in the different readout sectors. It is very well known that the radiation produces defects in the silicon crystal, which result in new energy states in between the conduction band and the valence band. These defects cause an increase of the leakage current since the number of thermally produced electron-hole pairs also increases. The increase of the leakage current is proportional to the fluence:

$$
\Delta I_{\text {leak }}=\alpha \cdot \Phi_{e q} \cdot V
$$

where $\alpha=4 \times 10^{-17} \mathrm{~A} /($ particle $\cdot \mathrm{cm})$ is the damage constant for $1-\mathrm{MeV}$ neutrons [4], $\Phi_{\text {eq }}$ is the average $1-\mathrm{MeV}$ neutron equivalent fluence, and $\mathrm{V}$ is the detector volume from which leakage currents are collected. The value of $\alpha$ is taken from [5] where first studies of the expected radiation damage in the TT are reported. A value of $\mathrm{T}=20^{\circ} \mathrm{C}$ is assumed in that report. Since the working average temperature of the sensors in the TT was around $13^{\circ} \mathrm{C}$, and the current scales with the operating temperature [6], a factor of 0.5 has to be applied to correlate the $I_{\text {leak }}$ in the note with the actual measurements in the TT. Thus, from $I_{\text {leak }}$ and the sensor geometry, the $\Phi_{\mathrm{eq}}$ can be extrapolated. Fig. 10 shows the results of the extrapolated (left picture) and the expected (from simulations done with Fluka) radiation flux in 1-MeV neutron 
equivalent. This study was performed with 2010 data and as can be seen in Fig. 10 there is a good agreement with the respect to simulations.

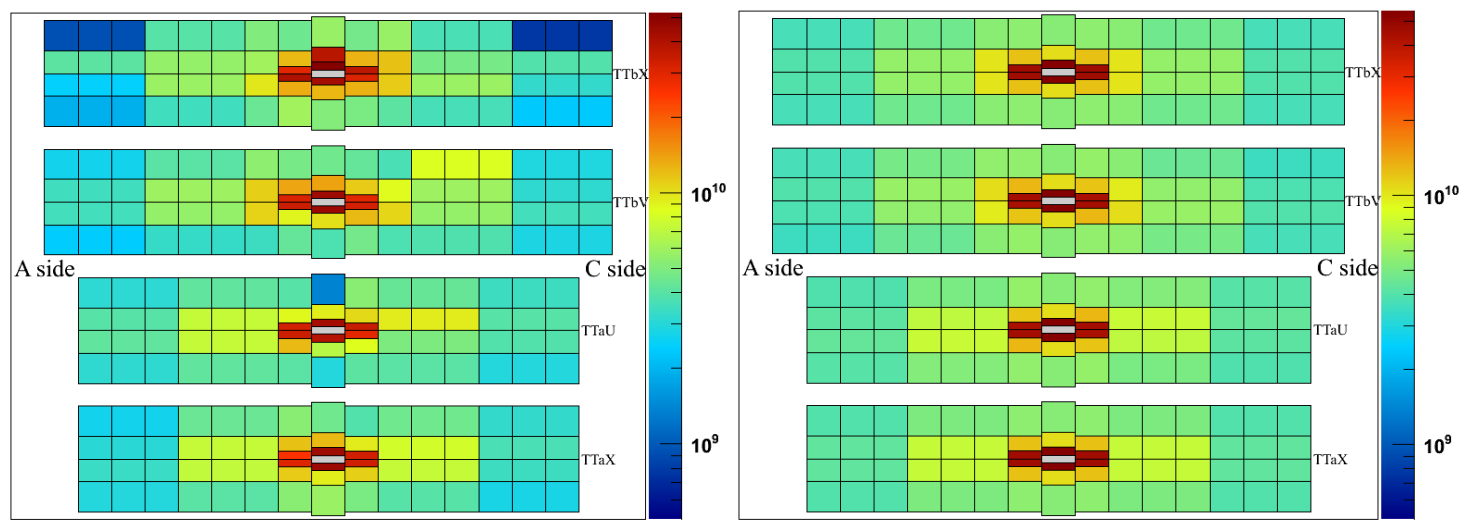

Figure 10: Radiation flux estimation in the TT comparing extrapolation from real data (left) vs. MC (right).

\section{Summary}

The detector was installed in summer 2008. Since then it has been running reliably and without major problems during the commissioning phase and physics data taking. Currently more than $99 \%$ of the detector channels are functional. The LHC proton-proton collision data has been used to tune different parameters of the detector, namely: perform the time alignment with an accuracy of $1 \mathrm{~ns}$; the signal to noise ratio was found to be in the range 12 to 15 for different strip lengths in the TT and $\sim 16.5$ and $~ 17.5$ for the the long and short IT ladders respectively; spatial alignment of the detector was done and currently an alignment precision of $\sim 11 \mu \mathrm{m}$ for IT compared to $\sim 18 \mu \mathrm{m}$ in TT is achieved; the hit resolution is $\sim 58 \mu \mathrm{m}$ and $\sim 62 \mu \mathrm{m}$ for IT and TT respectively, worse in data compared to simulation, but hints of the origin of this discrepancy are already in sight; the hit efficiency measured using high momentum tracks is around $99.65 \%$ in IT and $99.3 \%$ in TT; and finally the radiation damage agrees well with the expectations.

\section{References}

[1] The LHCb collaboration, The LHCb Detector at the LHC. Journal of Instrumentation, 2008 JINST 3 S08005

[2] J.-L. Agram et al., The silicon sensors for the Compact Muon Solenoid tracker - design and qualification procedure, NIM A, Volume 517, Issues 1-3, 2004, Pages 77-93.

[3] W. Hulsbergen, The Global covariance matrix of tracks fitted with a Kalman filter and an application in detector alignment, Nucl. Inst. Meth. A600, 471-477 (2009).

[4] M. Moll et al., Radiation hardness of silicon detectors - a challenge from high-energy physics, Nucl. Instr. Meth. A426 (1999) 87.

[5] M. Siegler, F. Lehner, M. Needham, O. Steinkamp, Expected Particle Fluences and Performance of the LHCb Trigger Tracker, LHCb-2004-070, September 6, 2004.

[6] S. Eidelman et al., Phys. Lett. B592 (2004) 1; http://pdg.lbl.gov/ 